\title{
RECONSTRUCTION OF 3D DEFORMATION FROM 2D MR VELOCITY MAPPING WITH INCOMPRESSIBILITY CONSTRAINTS
}

\author{
J. X. Gao' ${ }^{1}$, S. Masood ${ }^{1}$, F. Deligianni ${ }^{1}$, G. Z. Yang ${ }^{1,2}$ \\ ${ }^{1}$ Royal Society/Wolfson Foundation Medical Image Computing Laboratory \\ ${ }^{2}$ National Heart and Lung Institute \\ Imperial College of Science, Technology and Medicine, London, UK
}

\begin{abstract}
This paper presents a new method for calculating 3D myocardial deformation from multislice $2 \mathrm{D}$ magnetic resonance velocity mapping. The method first involves the rectification of in-plane velocity distribution with a variational vector restoration method. This restored $2 \mathrm{D}$ velocity is then used to estimate the through-plane velocity component by applying a local incompressibility constraint. A global optimization procedure was then used to derive the velocity distribution that conforms to the incompressibility constraint. The proposed method was validated by using a simulation phantom with different levels of noise. The derived velocity field permits a full 3D deformation analysis of the myocardium.
\end{abstract}

Keywords - Velocity mapping, myocardial deformation, strain rate, incompressibility.

\section{INTRODUCTION}

Measurement of regional cardiac contraction has long been thought to be of clinical significance. Being able to determine contraction abnormalities could help clinicians plan and direct treatment. There are several methods for assessing cardiac motion using Magnetic Resonance (MR). The two most common techniques are MR tagging and MR velocity mapping[1]. MR tagging is limited by the complex post-processing needed and by the fact that only apparent inplane motion is measured. The recent development of harmonic phase imaging (HARP) has reduced processing time considerably, from hours to seconds[2]. However, as with conventional tagging, only the in-plane motion can be measured. Velocity mapping provides a direct quantitative measurement of myocardial motion over time. However, this technique is limited by the acquisition time needed to acquire a full three-directional velocity data set. This is due to the fact that fully $3 \mathrm{D}$ velocity encoded data set requires at least four images, where one is the reference image and the other three are the velocity encoded images. Acquisition of cardiac motion is further complicated by respiratory motion, which superimposes a periodic motion due to the movement of the diaphragm. To overcome this problem, images are usually acquired in a single breath-hold. Hence, time available to acquire images becomes limited and only the two in-plane velocity components can be recorded with sufficient resolution and accuracy.

From these tagged or velocity images, myocardial deformation, i.e., strain and/or strain rate field can be obtained which are of importance in the diagnoses of cardiovascular diseases [3, 4]. However, as mentioned earlier, only the in-plane strain and/or strain rate is usually available. To analyze the real 3D deformation behavior of the heart, it is crucial to obtain all the three velocity components so that the $3 \mathrm{D}$ strain or strain rate field can be calculated. Another factor limiting the analysis of the true motion of the heart is the through-plane motion which will cause the myocardial tissue being imaged to move out of the imaging plane. As a result, the in-plane velocity data at different time frames will effectively correspond to different myocardial muscle. In this case, the subsequent direct computing of the strain or strain rate fields becomes inappropriate, and a through-plane motion correction is required.

In this paper, we describe a new approach to tackle the difficulties in assessing 3D myocardial strain and strain rate behavior from dense $2 \mathrm{D}$ velocity distributions such as those derived from HARP imaging. It is based on the mass conservation constraint, which leads to a generalized optimization process for the original velocity field. It minimizes the weighted residual error composed of the volume changes and the velocity fluctuation between the optimized and original velocity distribution. The throughplane velocity component is then reconstructed, and the $3 \mathrm{D}$ strain or strain rate distribution over time can be computed. A set of simulation dataset was generated to test the proposed method. The robustness of the method was tested by adding different levels of noise to the simulated in-plane velocity components. Preliminary results showed that the 3D velocity field is reconstructed accurately and robustly. However, the 3D strain field is prone to error due to noise as this requires integration over time. Further validation must be performed to extend this method into practical application.

\section{PINCIPLE AND METHOD}

The in-plane Lagrange strain rate tensor components $\left(\dot{\varepsilon}_{x}, \dot{\varepsilon}_{y}, \dot{\varepsilon}_{x y}\right)$ can be calculated as

$$
\begin{aligned}
& \dot{\varepsilon}_{x}=\frac{\partial \dot{u}}{\partial x}+\frac{1}{2}\left\{\left(\frac{\partial \dot{u}}{\partial x}\right)^{2}+\left(\frac{\partial \dot{v}}{\partial x}\right)^{2}\right\} \tau \\
& \dot{\varepsilon}_{y}=\frac{\partial \dot{v}}{\partial y}+\frac{1}{2}\left\{\left(\frac{\partial \dot{u}}{\partial y}\right)^{2}+\left(\frac{\partial \dot{v}}{\partial y}\right)^{2}\right\} \tau \\
& \dot{\varepsilon}_{x y}=\frac{1}{2}\left(\frac{\partial \dot{u}}{\partial y}+\frac{\partial \dot{v}}{\partial x}\right)+\frac{1}{2}\left(\frac{\partial \dot{u}}{\partial x} \frac{\partial \dot{u}}{\partial y}+\frac{\partial \dot{v}}{\partial x} \frac{\partial \dot{v}}{\partial y}\right) \tau
\end{aligned}
$$

where $(\dot{u}, \dot{v})$ are the velocity component along $x$ and $y$ axis, respectively, $\tau$ is the time interval between two successive frame.

The above calculation uses the directly measured velocity data $(\dot{u}, \dot{v})$. When there is significant through-plane motion during data acquisition the imaged mass layer will not correspond to the same myocardial tissue over time. A 
proper calculation should compensate for this through plane motion, i.e., to substitute the real velocity components computed by interpolating the velocity data between two adjacent slices that cover the given image plane. Thus, the through-plane velocity component $\dot{w}$ is needed even when only the in-plane deformation is of interest.

Incompressibility is an ideal state of a material, either solid or liquid, under any stress conditions. In reality, the myocardial muscle can be compressed to a certain extent as the coronary blood flow changes during the cardiac cycle. However, the actual volume change due to the compressibility of the material can be regarded to be negligible as compared to the apparent volume change produced by the inconsistent velocity distribution. This means that the error in computing deformation behavior by assuming incompressibility is always less than that caused by noise in the original velocity dataset. This is the basis of our approach to reconstruct 3D velocity field from 2D MR velocity mapping.

\section{A. $2 D$ Velocity Restoration.}

De-noising and restoration of the in-plane velocity fields can have a significant impact on the quality of the subsequent estimate of through-plane velocity. The technique used here for vector restoration is based on Chan and Shen's theoretical model[5]. This framework provides a systematic way for constructing the energy function, which is an extension of the classical total variational (TV) model. Coulon et al[6] apply the restoration of orientation features to the discrete case, i.e., the restoration of the diffusion field in diffusion tensor MRI.

The orientation feature $f$, in this case the velocity direction, is a non-flat, non-Euclidean feature, that lives on the unit circle $S^{1}$ and thus, is fully described by the angle $\vartheta$. The TV fitted energy is described as:

$$
\varepsilon^{T V}(f ; \lambda)=\sum_{\alpha \in \Omega_{n}} e(f ; \alpha)+\lambda \sum_{\alpha \in \Omega_{n}} \frac{1}{2} d_{l}{ }^{2}\left(f_{\alpha}{ }^{(0)}, f_{a}\right)
$$

where $d_{l}$ is the Euclidean distance, $f^{(0)}$ is the original image feature, $f_{\alpha}$ is the direction at voxel $\alpha$ and $e(f ; \alpha)$ is the strength function at voxel $\alpha$. The strength function is a measure of smoothness of the direction map and is defined as:

$$
e(f ; \alpha)=\left[\sum_{\beta \in N_{a}} d_{l}^{2}\left(f_{\beta}, f_{a}\right)\right]^{\frac{1}{2}}
$$

where $N_{\alpha}$ is a neighbourhood of $\alpha$. For a typical internal cross node $\alpha=(i, j)$ in image processing its neighbours are $\beta=(i, j-1),(i, j+1),(i-1, j),(i+1, j)$. Minimisation of Eq. 2 is described by a partial differential equation, which leads to the below relatively simple iterative scheme:

$$
\overline{g_{a}{ }^{n+1}}=\overline{f_{a}{ }^{n}}+\Delta t \cdot \prod_{f_{a}}\left(\sum_{\beta \in N_{a}} w_{a}{ }^{\beta} \overline{f_{\beta}}+\lambda \overline{f_{a}^{(0)}}\right)
$$

$$
\overline{f_{a}^{n+1}}=\frac{\overline{g_{a}{ }^{n+1}}}{\left\|g_{a}^{n+1}\right\|}
$$

where $\overline{f_{a}^{(i)}}$ the orientation feature, in our case the direction of the velocity, parameterised by the angle $\vartheta$ and the direction feature $\overline{f_{\alpha}}$ is restored using its neighbours $\overline{f_{\beta}}$, where $\beta \in N_{a}$. The restoration takes place only for the direction of vector fields while their lengths remain the same. Using this iterative scheme the restored velocity fields can be calculated within seconds even for a large number of iterations.

\section{B. Through-Plane Velocity Extraction.}

For large deformation, the incompressibility condition can be expressed by the Jacobian matrix such that its determinant always equals to unity:

$$
\left|\begin{array}{l}
1+\frac{\partial \dot{u}}{\partial x} \tau, \frac{\partial \dot{u}}{\partial y} \tau, \frac{\partial \dot{u}}{\partial z} \tau \\
\frac{\partial \dot{v}}{\partial x} \tau, 1+\frac{\partial \dot{v}}{\partial y} \tau, \frac{\partial \dot{v}}{\partial z} \tau \\
\frac{\partial \dot{w}}{\partial x} \tau, \frac{\partial \dot{w}}{\partial y} \tau, 1+\frac{\partial \dot{w}}{\partial z} \tau
\end{array}\right|=1
$$

Theoretically, the through-plane motion $\dot{w}$ can be deduced from the above equation provided the in-plane velocity field and a boundary condition are given. However, in reality, this is a very complicated computation process. If we focus on a small region (element), we can simplify the Jacobian determinant, hence to get an explicit expression of $\dot{w}$. In this case, we assume the through plane velocity is independent of $\mathrm{x}$ and $\mathrm{y}$, i.e.,

$$
\frac{\partial \dot{w}}{\partial x}=\frac{\partial \dot{w}}{\partial y}=0
$$

Then we can get:

$$
\dot{w}=-\frac{\frac{\partial \dot{u}}{\partial x}+\frac{\partial \dot{v}}{\partial y}+\frac{\partial \dot{u}}{\partial x} \frac{\partial \dot{v}}{\partial y} \tau-\frac{\partial \dot{u}}{\partial y} \frac{\partial \dot{v}}{\partial x} \tau}{1+\frac{\partial \dot{u}}{\partial x} \tau+\frac{\partial \dot{v}}{\partial y} \tau+\frac{\partial \dot{u}}{\partial x} \frac{\partial \dot{v}}{\partial y} \tau^{2}-\frac{\partial \dot{u}}{\partial y} \frac{\partial \dot{v}}{\partial x} \tau^{2}} \cdot z+\dot{w}_{0}
$$

where $\dot{w}_{0}$ is a constant determined by the boundary condition, or specifically, the velocity at the starting slice. In practice, this constant has no effect on the strain rate computation.

\section{3D Velocity Field Optimization.}

It is obvious that the through-pane velocity calculated based on a regional incompressibility constraint will fluctuate from element to element, bearing certain deviation with regard to actual distribution. Hence, we then perform a further optimization process which takes into account all the three velocity components. This is to minimize the overall cost function $\chi$, defined as:

$$
\chi=(1-\lambda) \sum \text { error }+\lambda \sum|\theta|
$$


where $\lambda$ is a factor weighting the conventional error term of the velocity field and the corresponding volume changes $\theta$. The error term is the normalized sum of square difference of in-plane velocity components $(\dot{u}, \dot{v})$ between the optimized and original values. It needs to be normalized so that it can be evaluated with the sum of volume change $\theta$, which has the general form of:

$$
\theta=\left|\begin{array}{l}
1+\frac{\partial \dot{u}}{\partial x} \tau, \frac{\partial \dot{u}}{\partial y} \tau, \frac{\partial \dot{u}}{\partial z} \tau \\
\frac{\partial \dot{v}}{\partial x} \tau, 1+\frac{\partial \dot{v}}{\partial y} \tau, \frac{\partial \dot{v}}{\partial z} \tau \\
\frac{\partial \dot{w}}{\partial x} \tau, \frac{\partial \dot{w}}{\partial y} \tau, 1+\frac{\partial \dot{w}}{\partial z} \tau
\end{array}\right|-1
$$

This optimization process will lead to a further improved $3 \mathrm{D}$ velocity field. The weighting factor will however affect the result, for example, $\lambda=0$ will make the final optimized velocity return to the original measured value. The optimal $\lambda$ was found to be around $0.6-0.8$. From the improved 3D velocity field, we can calculate the complete 3D deformation components, i.e. the strain rate field.

\section{SiMULATION DATASET}

To test the proposed method, a 3D simulation dataset was produced which corresponded to a cylindrical structure. All three velocity components were simulated and the incompressibility constraint was incorporated. This dataset aims to simulate the actual cardiac motion and the real MR imaging setup, where the imaging planes are fixed while the mass points imaged are moving throughout the imaging process.

The velocity components are:

$$
\begin{aligned}
& \dot{u}=K_{x}(x, y) \cos \varpi t \\
& \dot{v}=K_{y}(x, y) \cos \varpi t \\
& \dot{w}=K_{z}(x, y, z) \cos \varpi t
\end{aligned}
$$

The displacement components are the summation of velocity components for the same mass point over time:

$$
\begin{aligned}
& u=K_{x}(x, y) \frac{\sin \varpi t}{\sigma} \\
& v=K_{y}(x, y) \frac{\sin \varpi t}{\sigma} \\
& w=K_{z}(x, y, z) \frac{\sin \varpi t}{\sigma}
\end{aligned}
$$

In the above equations, the in-plane kernel functions, $K_{x}$ and $K_{y}$, are predefined, and the z-component kernel function $K_{z}$ is calculated from

$$
K_{z}(x, y, z)=-\frac{\frac{\partial K x}{\partial x}+\frac{\partial K y}{\partial y}+\frac{\partial K x}{\partial x} \frac{\partial K y}{\partial y}-\frac{\partial K x}{\partial y} \frac{\partial K y}{\partial x}}{1+\frac{\partial K x}{\partial x}+\frac{\partial K y}{\partial y}+\frac{\partial K x}{\partial x} \frac{\partial K y}{\partial y}-\frac{\partial K x}{\partial y} \frac{\partial K y}{\partial x}} \cdot z
$$

Now the problem of simulation becomes, given a current imaging point (mass point) $P_{t}(x, y, z)$, to find its original position $P_{t 0}\left(x_{0}, y_{0}, z_{0}\right)$. It is the attribute of the original point $P_{t 0}$ that determines the validity of the current imaging point $P_{t}$, i.e., whether this current point has a valid velocity value or not. If its retrospective original point was located within a predefined mass region, then the current imaging point is valid. Otherwise, the current mass point is coming from a virtual original mass point that is outside the predefined mass region, so it is no longer a valid mass point. This forms an inverse search problem, i.e., at the current mass point $P_{t}(x, y, z)$, find its original point $P_{t 0}\left(x_{0}, y_{0}, z_{0}\right)$, such that

$$
\begin{aligned}
& x=x_{0}+u\left(x_{0}, y_{0}, z_{0}\right) \\
& y=y_{0}+v\left(x_{0}, y_{0}, z_{0}\right) \\
& z=z_{0}+w\left(x_{0}, y_{0}, z_{0}\right)
\end{aligned}
$$

A dataset consisting of 6 slices with a complete cycle of 16 frames was generated.

\section{RESULTS}

The in-plane velocity components of the 3D simulation dataset are put into our 3D deformation analysis system. Noise levels of $10 \%$ and $25 \%$, which correspond to SNR of $22.56 \mathrm{~dB}$ and $14.61 \mathrm{~dB}$, were added to test the robustness of the algorithm. Figure 1 shows the reconstructed throughplane velocity component distribution at slice 3 as compared to the actual distributions, where slice 1 is the starting slice, i.e., its though-plane motion is zero in the entire imaging plane. It is clear that the overall trend of the through-plane velocity distribution can always be restored even with noise level as high as $14.61 \mathrm{~dB}$. This provides the possibility of applying overall interpolation so that an even more accurate estimation of the spatial derivative information can be obtained.

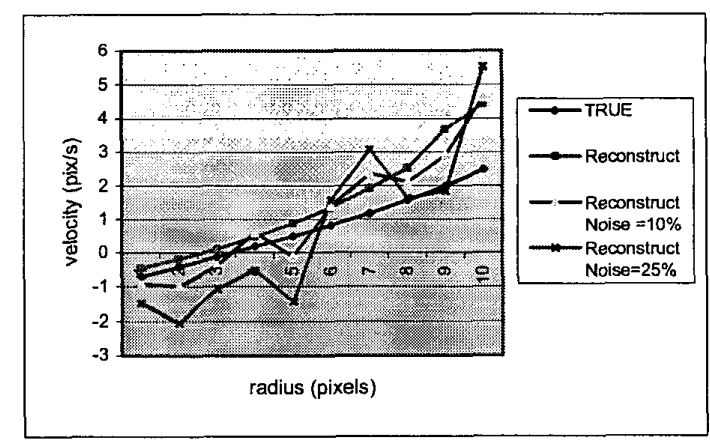

Fig. 1. Reconstructed through-plane velocity distribution. A low level of noise, less than $10 \%(22.56 \mathrm{~dB})$, ensures that the result is robustly reconstructed. A noise level of $25 \%(14.61 \mathrm{~dB})$ or greater will cause the velocity reconstruction to be prone to error. 

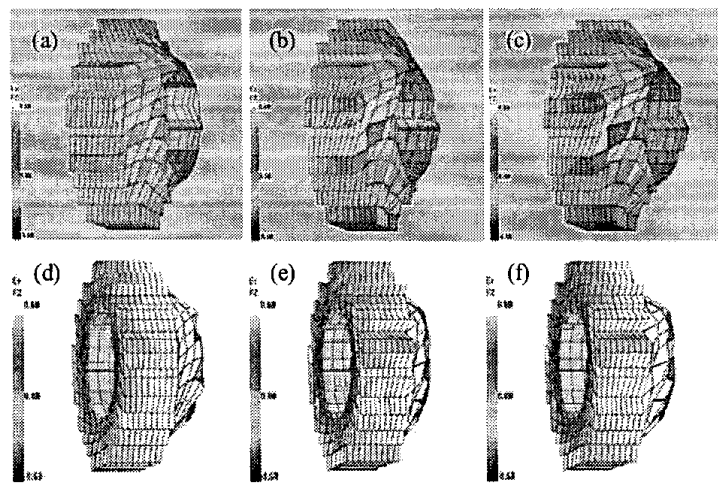

Fig. 2. 3D color display of reconstructed circumferential (upper row) and radial (lower row) strain rate field. (a) and (d): Real strain rate distribution. (b) and (e): The final reconstructed 3D strain rate distribution with optimization of $10 \%$ noise $(22.56 \mathrm{~dB})$ added dataset. (c) and (f): Directly reconstructed result based on local incompressibility constraint without optimization, also with $10 \%$ level of noise $(22.56 \mathrm{~dB})$

Fig. 2 shows the 3D distribution of circumferential and radial strain rate field at frame 4 of the dataset. The original distribution, a dataset with $10 \%$ noise reconstructed with and without optimization are displayed. It can be seen from Figure 2 (b) and (e) that incorporating the optimization improves the strain rate result. The reconstructed 3D velocity field is integrated to produce the $3 \mathrm{D}$ displacement field for visualization of the deformed 3D shape.

\section{DISCUSSION AND CONCLUSION}

It is worth noting that the incompressibility constraint used in this paper can only be used to directly reconstruct the derivative of the through-pane motion. The complete reconstruction of the 3D deformation field requires an appropriate selection of the boundary condition, i.e., the initial motion at the boundary surface. For tracking material over time, it is important to ensure that the displacement associated with the tissue is accurately derived. For analyzing myocardial contractility, the basal plane defined by the mitral valve can be used for this purpose.

For calculating the strain rate distribution, however, it is reasonable to use the reconstructed $3 \mathrm{D}$ velocity field directly without through-plane motion correction. In contrast, the computation of strain field distribution is more prone to noise as involves the accumulation of the rate of displacement. The main purpose of the proposed method is for deriving 3D velocity distribution from densely mapped $2 \mathrm{D}$ velocity distributions such as those obtained from HARP imaging such that through plane contraction can be taken into account. It must be noted, however, there are specific artifacts that can be introduced in the velocity maps derived from HARP imaging, which could lead to significant errors in in-plane velocity distributions. The use of improved imaging sequence design such as CSPAMM can alleviate these problems, but these artifacts must be carefully addressed if the proposed method is to be used for patient studies involving HARP imaging.

In summary, we have demonstrated that it is possible to derive the through-plane velocity component by using the incompressibility constraint and we have demonstrated its potential in a numerical phantom experiment. The proposed method has been implemented through a three step process: (1) $2 \mathrm{D}$ velocity restoration, (2) through-plane velocity component reconstruction based on local incompressibility, and (3) overall optimization of the reconstructed 3D velocity field. Preliminary results show that the method works well in reconstructing the $3 \mathrm{D}$ strain rate field in a simulated environment. However, further in vivo validation is needed to assess the robustness and accuracy of the algorithm for patient studies.

\section{REFERENCES}

[1] S. Masood, G. Z. Yang, D. J. Pennell, D. N. Firmin, "Investigating intrinsic myocardial mechanics: the role of MR tagging, velocity phase mapping, and diffusion imaging," J. Magn. Reson. Imaging, vol. 12, pp. 873-83, 2000

[2] N.F. Osman, E.R. McVeigh, and J.L. Prince, "Imaging heart motion using harmonic phase MTI", IEEE Trans. Med. Imaging. vol. 19, pp. 186-202, March 2000.

[3] Y. Zhu, M. Drangova, N. J. Pelc, "Estimation of deformation gradient from cine-PC velocity data," IEEE Trans. Med. Imaging, vol. 16, pp. 840-51, 1997.

[4] E. McVeigh, C. Ozturk, "Imaging myocardial strain," IEEE Signal Processing Magazine, vol.18, pp. 44 -56, 2001.

[5] Tony Chan and Jianhong Shen, 'Variational Restoration of Non-Flat Image Features: Models and Algorithms', SIAM Journal on Applied Mathematics, 2000.

[6] O. Coulon, D.C. Alexander and S.R.Arridge, 'Tensor Field Regularisation for DT-MR Images', Proc. MIUA 2001, Medical Image Understanding and Analysis, Birmingham, UK, 2001 